\title{
Hidden Markov Model Forward Algorithm
}

National Cancer Institute

\section{Source}

National Cancer Institute. Hidden Markov Model Forward Algorithm. NCI Thesaurus.

Code C81018.

A Hidden Markov Model algorithm that calculates recursively forward along the observation sequence. 